\title{
La equidad en el aprendizaje de profesores mediante la interacción entre experiencia y competencia
}

Equity in Teacher Learning Through the Interaction between Competence and Experience

A equidade na aprendizagem de professores por meio da interação entre experiência e competência

\section{Avenilde Romo-Vázquez* (iD orcid.org/0000-0002-1364-5997 Ana Luisa Gómez-Blancarte** (iD) orcid.org/0000-0002-8837-8643}

\footnotetext{
Artículo de investigación

Revista Colombiana de Educación, N.7 74. primer semestre de 2018, Bogotá, Colombia.

Para citar este artículo: Romo, A. y Gómez, A. (2018). La equidad en el aprendizaje de profesores mediante la interacción entre experiencia y competencia. Revista Colombiana de Educación, (74), 269-287.
}

\section{(c) $(1) \Theta$}

* Doctora en Didáctica de las Disciplinas. Profesora-investigadora del Programa de Matemática Educativa del Centro de Investigación en Ciencia Aplicada y Tecnología Avanzada del Instituto Politécnico Nacional. Ciudad de México, México. Correo electrónico: aromov@ipn.mx

** Doctora en Ciencias en la Especialidad de Matemática Educativa. Profesora-investigadora del Programa de Matemática Educativa del Centro de Investigación en Ciencia Aplicada y Tecnología Avanzada del Instituto Politécnico Nacional, Ciudad de México, México. Correo electrónico: algomezb@ipn.mx 


\title{
Resumen
}

Se presenta un estudio sobre la instrucción que se ofrece en un programa de profesionalización de profesores de matemáticas del Centro de Investigación en Ciencia Aplicada y Tecnología Avanzada del Instituto Politécnico Nacional de México en la modalidad en línea y a distancia. El estudio se enmarca en la teoría de comunidades de práctica (CoP) propuesta por Wenger $(1998,2010)$, y en particular en las nociones de experiencia y competencia. Se analizan las interacciones de los profesores durante su participación en una unidad de aprendizaje acerca de los recursos didácticos del y para el profesor de matemáticas. El análisis se centra en la interacción entre la experiencia de los profesores y la competencia que los educadores promueven para alinear esta experiencia. Se concluye que la alineación entre la experiencia de los profesores y la competencia representada por los educadores es un elemento indispensable para fomentar la equidad en el aprendizaje de los profesores.

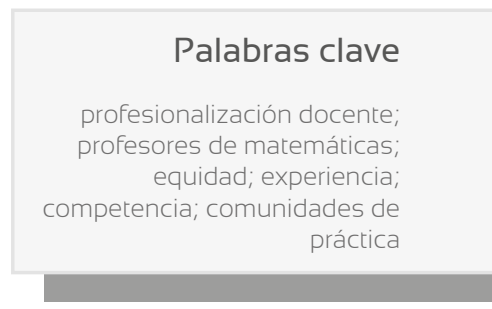

\section{Keywords}

teacher professionalization; math teachers; equity; experience; competence; communities of practice

\begin{abstract}
In this paper, we present a study on the instruction offered in a professionalization program for math teachers from the online and distance programs of the Center for Research and Advanced Studies of the National Polytechnic Institute in Mexico. The study is based on Wenger's $(1998,2010)$ theory of communities of practice (CoP), and particularly on the concepts of competence and experience. We analyze the teachers' interactions during their participation in a learning unit on the didactic resources of and for math teachers. The analysis focuses on the interaction between the teachers' experience and the competence promoted by educators in order to align that experience. We conclude that the alignment between the teachers' experience and the competence that they represent is essential to promote equity in teachers' learning.
\end{abstract}

\section{Resumo}

Apresenta-se um estudo sobre a instrução oferecida em um programa de profissionalização de professores de matemáticas no Centro de Pesquisa em Ciência Aplicada e Tecnologia Avançada do Instituto Politécnico Nacional do México na modalidade online e a distância. O estudo inscreve-se na teoria das comunicações de prática (CoP) proposta por Wenger $(1998,2010)$, e, particularmente, nas noçōes de experiência e competência. Analisam-se as interações dos professores durante sua participação em uma unidade de aprendizagem acerca dos recursos didáticos para o professor de matemáticas. A análise está centrada na interação entre a experiência dos professores e a competência que os educadores promovem para alinhar essa experiência. Como conclusão, o alinhamento entre a experiência dos professores e a competência representada pelos educadores é um elemento indispensável para fomentar a equidade na aprendizagem dos professores.

\section{Palavras-chave}

profissionalização docente; professores de matemáticas; equidade; experiência; competência; comunidades de prática 


\section{Profesionalización de profesores de matemáticas en línea: un proyecto de equidad}

El Programa de Matemática Educativa (Prome) del Centro de Investigación en Ciencia Aplicada y Tecnología Avanzada del Instituto Politécnico Nacional de México (Cicata-IPN) se fundó en el año 2001. Su principal objetivo era ofrecer una formación didáctica a profesores de matemáticas en servicio, en un principio, de México y, posteriormente de Latinoamérica, en la modalidad en línea y a distancia. El propósito inicial del Prome fue atender a aquellos profesores de matemáticas mexicanos que deseaban especializarse en la disciplina de la educación matemática a través del estudio en posgrados (maestría y doctorado), pero que por diferentes motivos (laborales, personales, económicos, geográficos, institucionales) no podían cumplir con los requerimientos de ingreso de otros programas de posgrado presenciales. Superando las expectativas, al lanzar su primera convocatoria de ingreso, el Prome no solo recibió solicitudes de profesores mexicanos, sino también de profesores de matemáticas de otras regiones de Latinoamérica, como Argentina, Brasil, Chile, Colombia, Costa Rica, Cuba, Guatemala, Perú, Uruguay, Ecuador y Venezuela. El hecho de que profesores de otros países latinoamericanos solicitaran su ingreso al Prome mostró la necesidad de ofrecer programas que tomaran en cuenta, principalmente, la situación laboral de los profesores pues, de acuerdo con Moreno (2003), en México, los profesores que estudian un posgrado lo hacen simultáneamente con su trabajo docente. Esta situación no era exclusiva de los profesores mexicanos.

A través de la modalidad en línea y a distancia, el Prome se convertía en una opción para los profesores ubicados geográficamente en zonas alejadas de las universidades, los que son padres o madres de familia y para aquellos que buscaban formarse sin dejar de ser profesores en ejercicio. Con el paso del tiempo, el proyecto de formación se fue consolidando y al mismo tiempo innovando. Por una parte, se iba ganando conocimiento sobre los saberes, expectativas y necesidades del profesor de matemáticas; por otra parte, la evolución en las tecnologías informáticas posibilitaba nuevas formas de comunicación: compartir un video, realizar una videoconferencia, habilitar un foro, compartir archivos, artículos, actividades didácticas, fotos, gráficas, ecuaciones. Estas formas de comunicación eran cada vez más sencillas de realizar y en un menor tiempo. Todo esto influyó en la forma de diseñar las unidades de aprendizaje, de proponer tareas de investigación, de experimentación en el aula y de reflexión conjunta (debate en grupos pequeños y grandes). 
Los estudiantes del Prome son profesores de matemáticas en ejercicio de distintos lugares de México y de Latinoamérica con diferentes formaciones académicas: algunos de profesor de matemáticas, otros de ingeniería, economía, actuaria o carreras que incluyen un gran número de asignaturas de matemáticas. Asimismo, ejercen su labor docente en niveles educativos que van desde la secundaria hasta la universidad $y$, en algunos casos, la primaria. Las diferencias culturales, geográficas, institucionales, de conocimiento (matemático y pedagógico), de experiencia docente y de nivel educativo en el que enseñan los profesores exigen aprendizajes distintos. En este sentido, conforman un grupo heterogéneo, lo que representa un gran reto para concebir e implementar una instrucción de equidad.

De acuerdo con Weissglass (1997, citado en Sowder, 2007) la equidad es un proceso continuo por el cual incrementamos nuestra capacidad y compromiso con nosotros mismos y con la sociedad para, entre otros, proporcionar los recursos necesarios para asistir a las personas en su aprendizaje. Desde este punto de vista, tomar en cuenta la equidad en un contexto de instrucción de profesores de matemáticas plantea al menos dos cuestiones importantes: (1) ¿Cómo diseñar unidades de aprendizaje que propicien los saberes y conocimientos que cada profesor requiere? Y (2) ¿Cómo tener en cuenta los conocimientos y resultados de la investigación en educación matemática para ponerlos a disposición de los profesores? Estas preguntas guían el estudio que presentamos en este documento, cuyo objetivo es analizar la manera en que la experiencia de los profesores y la competencia que los educadores promueven favorecen la equidad en el aprendizaje de los profesores. Para ello, mostramos el análisis de una Unidad de Aprendizaje (UA) concebida e implementada en este programa.

\section{El desarrollo profesional de los profesores: elemento clave en la educación matemática de equidad}

En la declaración sobre la ciencia y el uso del saber científico adoptada por la Conferencia mundial en Budapest de 1999, se manifiesta que el saber científico debe ser accesible a todos:

10. Que el acceso al saber científico con fines pacíficos desde una edad muy temprana forma parte del derecho a la educación que tienen todos los hombres y mujeres, y que la enseñanza de la ciencia es fundamental para la plena realización del ser humano, para crear una capacidad científica endógena y para contar con ciudadanos activos e informados, [...] (Organización de las Naciones Unidas para la Educación, la Ciencia y la Cultura,unesCo, 1999, punto 10). 
Lograr que los saberes matemáticos sean efectivamente un derecho de todo ciudadano requiere de programas de formación docente apropiados, en el sentido de Artigue (2011):

Una formación apropiada debería, en particular, ayudar eficazmente a los profesores a elaborar tareas de investigación, susceptibles de permitir actividades de investigación matemáticamente productivas en concordancia con las condiciones de la clase, ayudarles a jugar el rol de guías y de mediadores, para gestionar estas actividades de manera matemáticamente eficaz. (p. 22).

En cuanto a la disciplina, Sowder (2007) reconoce seis objetivos que deberían ser atendidos en el desarrollo profesional de los profesores de matemáticas: (1) una visión compartida de la enseñanza y el aprendizaje de las matemáticas, (2) un entendimiento de las matemáticas para el nivel enseñado, (3) un entendimiento de cómo los estudiantes aprenden matemáticas, (4) un profundo conocimiento pedagógico, (5) un entendimiento del rol de la equidad en la escuela de matemáticas y (6) un sentido de sí mismo como profesor de matemáticas. En el quinto objetivo, que resulta el de mayor interés para este documento, Sowder destaca cómo el currículo o plan de estudios "neutral" va en contra de mostrar el fuerte rol social de la educación matemática. De hecho, señala la existencia de un estereotipo según el cual las matemáticas son un conjunto de conocimientos destinados a grupos "privilegiados", y que una manera de romperlo es incluir en la enseñanza una diversidad de contextos de la vida real. Para ello, los profesores tendrían que ser sensibles a los procesos de producción y de difusión de las matemáticas, pero también a las formas en que estas se utilizan. Esto implica, como lo señala Doerr (2007), reconocer que el profesor de matemáticas no necesariamente está en posibilidad de conocer estos contextos de uso de las matemáticas. Además, los profesores deben estar preparados para enseñar matemáticas a poblaciones diversas, reconocer en ellas las necesidades o expectativas de sus estudiantes y tratar de atenderlas desde su enseñanza (Sowder, 2007).

Para tener en cuenta estas exigencias en el desarrollo profesional de profesores de matemáticas en ejercicio, en el Prome se diseñan UA basadas en paradigmas alternativos de enseñanza de las matemáticas, como el del cuestionamiento del mundo (Chevallard, 2013). Estos diseños se hacen reconociendo las necesidades particulares de cada profesor y atendiéndolas en el proceso de instrucción, por lo que la equidad es uno de sus rasgos característicos. Para analizar la forma en que la equidad tiene lugar durante la implementación de las UA, consideramos como marco teórico las comunidades de práctica, una teoría social del aprendizaje que detallamos a continuación. 


\section{Comunidades de práctica: experiencia y competencia}

La teoría de comunidades de práctica (CoP) se utiliza cada vez más para estudiar el desarrollo profesional de profesores, tanto para entender como para favorecer el aprendizaje en contextos educativos (Wenger, 1998; Wenger, 2010; Helleve, 2010). De acuerdo con Wenger, las CoP son un lugar de aprendizaje tanto para los nuevos miembros como para los que ya pertenecen, pues es un contexto en el cual las nuevas ideas se transforman en conocimiento. Los profesores educadores (en adelante, educadores) y profesores estudiantes (en adelante, profesores) del Prome forman una CoP. Mientras que los educadores son miembros de pleno derecho en esta comunidad, los profesores son miembros periféricos, pues su participación no los compromete en las acciones y decisiones que los educadores toman para organizar la práctica del programa: profesionalizar a los profesores. La tarea de los educadores del Prome es organizar y guiar el aprendizaje de los profesores. Ellos crean condiciones para que, por un lado, los profesores participen en actividades que influyan en su práctica docente; por otro lado, tengan los recursos necesarios para coordinar esa participación. La tarea de los profesores es la producción y adopción de significados acerca de los recursos en torno a los cuales los educadores organizan su aprendizaje. En efecto, para Wenger (2001) "adoptar un significado es contribuir a su producción interactiva" (p. 246). La producción y adopción son un proceso de apropiación del conocimiento tanto para los profesores como para los educadores.

Las relaciones entre los educadores y profesores se fundamentan en lo que saben y conocen sobre la Educación Matemática. Es decir, en el compromiso que "se basa en lo que hacemos y conocemos, así como en [...] las contribuciones y el conocimiento de otros" (Wenger, 2001, p. 103). Desde este punto de vista, el Prome es un espacio de "encuentros limitáneos", donde los profesores y educadores intercambian conocimientos acerca de sus respectivas comunidades de práctica (Sztajn, Wilson, Edgington y Myers, 2014). Este intercambio configura el conocimiento experto que los profesores tienen en la práctica de enseñar matemáticas y los educadores en la práctica de hacer investigación en educación matemática y formar profesores. Dentro del Prome, en particular durante el desarrollo de las UA, los profesores están expuestos al conocimiento experto de los educadores, es decir a una competencia que está fuera de su práctica porque simplemente son miembros de una práctica distinta, donde han experimentado otras cuestiones de la educación matemática que los han hecho expertos en ello. Para que el aprendizaje tenga lugar durante las $\cup A$, es necesario que la experiencia del profesor sobre su práctica de enseñar matemáticas y la competencia de los educadores sobre la investigación en educación matemática y formación de profesores 
interactúen. Esto porque "dentro de una comunidad, el aprendizaje ocurre porque la competencia y la experiencia necesitan converger para que la comunidad exista" (Wenger, 2010, p. 126).

En la comunidad del Prome, los profesores personifican la experiencia y los educadores la competencia. Nuestra descripción de la experiencia de los profesores va más allá de un conocimiento meramente cognitivo, se trata de su vivencia como profesores de matemáticas. La experiencia a la que Wenger (2001) se refiere es de identidad, la cual "no es sólo una acumulación de detalles e información, sino también un proceso de llegar a ser, de convertirse en una persona determinada o, a la inversa, de evitar convertirse en determinada persona" (p. 260). En ese sentido, el conocimiento práctico del profesor, aquel que ha adquirido durante su práctica de enseñar, es reconocido como legítimo, pues es un elemento constitutivo de su experiencia y necesario para la comunidad del Prome. Sin embargo, cuando los profesores entran al programa, confrontan su experiencia con la competencia de los educadores para extender esa experiencia y reinterpretarla a través de un conocimiento colaborativo, usando los recursos informativos y las herramientas de representación de una amplia cultura (Wells, 2004).

Los educadores personifican el proceso de competencia porque son ellos quienes deciden qué recursos informativos y herramientas utilizar para alinear la experiencia de los profesores. Estas decisiones se basan en los resultados de la investigación en educación matemática y en la propia experiencia de los educadores como formadores de profesores. En este sentido, son los educadores quienes definen los criterios y expectativas por los cuales ellos reconocen cuándo la experiencia de los profesores encaja con el "régimen de competencia" definido por los educadores. En efecto, los criterios y expectativas son propios de cada comunidad, son lo que Wenger (2010) denomina "régimen de competencia". En términos generales, nuestro régimen de competencia incluye: un entendimiento por parte de los profesores de los recursos que les son proporcionados, su disposición por comprometerse de manera productiva con el resto de sus compañeros y con la comunidad misma, adoptar y emplear el repertorio de elementos teóricos que forman parte del discurso de esos recursos. Estos recursos son indispensables porque alinean el conocimiento práctico de los profesores, representan la competencia que ellos tienen que confrontar. En este sentido, la alineación es un proceso por el cual los educadores coordinan los significados que esos recursos demandan, de manera que los profesores logren las competencias esperadas. Wenger (2010) explica que la alineación es un proceso doble porque no es un mero consentimiento de conformidad o pasividad. Es decir, no se espera que los profesores apliquen a ciegas los recursos proporcionados, sino que contribuyan en la producción y adopción de sus significados. De hecho, Wenger (2001) aclara que mantener en interacción

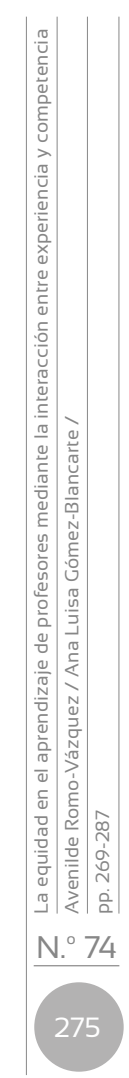


la producción y adopción es fundamental para el aprendizaje, ya que este "depende de nuestra capacidad de contribuir a la producción colectiva de significado porque es mediante este proceso por el que la experiencia y la competencia se impulsan mutuamente" (p. 247).

Así pues, en este estudio investigamos cómo interactúan la experiencia de los profesores y la competencia de los educadores para favorecer el aprendizaje que los profesores necesitan.

\section{Contexto del estudio}

El Prome ofrece dos programas de posgrado: Maestría en Ciencias y Doctorado en Ciencias, ambos en la especialidad de Matemática Educativa. El programa de maestría (dos años de duración) está enfocado en la profesionalización de profesores de matemáticas, mientras que el de doctorado (tres años de duración) tiene un enfoque centrado en la investigación. El presente estudio se centra en el programa de maestría.

La comunidad de educadores del Prome está formada por ocho profesores-investigadores en educación matemática. Aunque concurren en un mismo espacio físico, la interacción con los profesores es siempre no presencial. El único momento en que el profesor debe asistir a las instalaciones del Prome es el día de su examen de grado. Las actividades principales en torno a las cuales se organiza el Prome se pueden caracterizar en tres fases: (1) ingreso de los profesores, (2) instrucción y (3) proyectos de investigación. En cada una de estas es posible observar la relación entre la experiencia de los profesores y la competencia de los educadores. Este estudio se centra en la fase de instrucción.

\section{El proceso de instrucción en el Prome}

El Prome ofrece un plan de estudios que se compone de trece cursos: nueve UA, tres seminarios en matemática educativa y el trabajo de tesis. Durante los primeros tres semestres se ofrecen cuatro cursos (tres UA y un seminario) en cada uno; en el cuarto semestre, sólo el trabajo de tesis. Todas las UA y seminarios se colocan en la plataforma Moodle (véase http://cursos.cicata. edu.mx), su diseño y contenido varía según el (los) educador(es) responsable(s) y las necesidades de los profesores participantes. La metodología de las UA se puede clasificar en dos tipos: teóricas y teórico-prácticas.

» Teóricas. Se distinguen por incluir entre sus contenidos elementos de una o más herramientas teóricas o metodológicas asociadas a una(s) teoría(s) en educación matemática. Por medio de este tipo de UA se pretende dotar de competencias útiles para la gestión y regulación de la práctica docente, pero también para convertir la enseñanza en un objeto de investigación. 
» Teórico-prácticas. En estas UA se presentan elementos teóricos o metodológicos de una o varias teorías en educación matemática, con el objetivo de que estos provean a los profesores de competencias útiles para la práctica docente. Para el desarrollo de estas uA la experiencia docente de los profesores es fundamental.

La mayoría de las UA se ubican en las teórico-prácticas y su objetivo es crear un puente entre la investigación en educación matemática y el aula. Están organizadas en actividades que se cursan en cuatro o cinco semanas e incluyen actividades de investigación y de experimentación en el aula (en promedio una por semana). Las actividades de investigación son las más cercanas a las desarrolladas en la educación matemática: leer y hacer síntesis de un artículo, conocer y analizar herramientas teóricas, identificar herramientas metodológicas, generar explicaciones sobre dificultades de aprendizaje, analizar tanto el diseño didáctico de una asignatura como su implementación en el aula, etc. Las actividades de experimentación en el aula son prácticas: están centradas en diseñar una actividad de enseñanza, organizar un proceso de estudio, aplicar un diseño didáctico y detectar una problemática en el aula. El desarrollo de las actividades, tanto de investigación como de experimentación en el aula, se realizan o se comunican a través de las distintas herramientas de la plataforma Moodle (foros, wikis, archivos y videos compartidos en el espacio del curso), reuniones en Skype e intercambio de correos electrónicos. Todas estas herramientas favorecen la comunicación escrita y asíncrona, lo que permite, en casi todos los casos, tener respaldos electrónicos de la actividad, memoria de los intercambios y participaciones reflexivas. Al igual que las $\cup A$, los seminarios en educación matemática y el trabajo de tesis también son de corte teórico-práctico, pues se enfocan en el desarrollo del proyecto de investigación que se reporta en la tesis.

A fin de ilustrar cómo interactúan la experiencia del profesor y la competencia de los educadores en la fase de instrucción, se analizó una UA teórico-práctica: Procesos de Institucionalización de la Matemática Escolar (PIME), la cual se impartió del 24 de octubre al 18 de noviembre de 2016 y fue continuación de la UA Análisis del Discurso Matemática Escolar I (ADME ।) (impartida del 26 de septiembre al 21 de octubre de 2016). Los profesores en ambas uA fueron 20, ellos conforman la generación 2016 (11 mexicanos, 5 uruguayos, 1 ecuatoriano, 2 colombianos, 1 chileno). La experiencia docente de los 20 profesores en cuanto al nivel educativo en que impartían clases era diversa: un profesor impartía clases en el nivel secundaria (estudiantes de 13 a 15 años), 5 en el nivel bachillerato (estudiantes de 16 a 18 años), 6 en el nivel universitario (estudiantes de 19 a 22 años o más) y el resto impartían clases en dos o tres de los niveles mencionados. El tiempo de experiencia como profesor de matemáticas también variaba entre 1 y 30 años. 


\section{Diseño de la UA PIME}

Esta UA fue diseñada e impartida por dos educadores del Prome, y el diseño se motivó por el reconocimiento de una problemática docente: los profesores de matemáticas en ejercicio producen recursos didácticos para sus clases, pero no los comparten con otros colegas, por lo tanto pierden la oportunidad de generar una reflexión entre pares sobre las potencialidades y los efectos de esos recursos en la enseñanza. Así, el objetivo de esta uA fue poner a disposición de los profesores herramientas para analizar y producir recursos didácticos entre pares. Se consideró la investigación doctoral de Georget (2009), que aborda esta problemática. Para este autor, un recurso didáctico se concibe como la actividad o el conjunto de actividades didácticas que se acompañan de una guía para su implementación en el aula de matemáticas. La contribución de Georget es un conocimiento basado en la investigación; en tal medida es un medio de alineación porque permitía a los educadores confrontar la experiencia de los profesores en el diseño y evaluación de recursos didácticos con las competencias que demandaban las herramientas propuestas por Georget. La UA se organizó por medio de cuatro actividades principales.

» Actividad 1. Resolver y evaluar en pareja el recurso didáctico propuesto por otra pareja de profesores en una UA precedente (ADME 1). Se habilitó un foro para desarrollar esta actividad, en la que la experiencia de los profesores era el elemento clave.

» Actividad 2. Leer y analizar un documento producido por los educadores, basado en la tesis doctoral de Georget (2009), en el que se presentaban herramientas de análisis de los recursos didácticos. Participar en un foro en equipo de cuatro integrantes para discutir la lectura y ver un video en el cual Georget precisaba las herramientas de análisis . Este documento representa la competencia requerida para alinear el análisis de los recursos didácticos.

» Actividad 3. Se pidió a cada pareja elaborar una guía para su diseño didáctico, -producido en la actividad 4 del curso ADME I-. En la guía didáctica debían detallar las características de su diseño, su utilidad, utilizabilidad, adaptabilidad y cada uno de sus potenciales (herramientas presentadas en la lectura y video de la actividad 2). Además, se les indicó que era posible realizar nuevas modificaciones, basándose tanto en las observaciones que les hicieron sus compañeros en la primera actividad, como en las herramientas teóricas que se discutieron en la segunda. Esta actividad invitaba a los profesores a negociar la interpretación de las herramientas con su pareja y a producir un nuevo recurso (la guía) para hacer que su diseño didáctico fuera más comprensible y transferible al contexto del aula. 
» Actividad 4. En esta, se pidió a los profesores, primero, presentar el recurso diseñado a un profesor de matemáticas externo al curso y solicitarle que lo analizara y evaluara. El colega debía resolver las tareas propuestas en el recurso y luego evaluarlo, como experto en la enseñanza de la matemática. Además, se les pidió a los profesores que escribieran un reporte del trabajo realizado con el colega. Se habilitaron dos foros, uno para compartir dudas relacionadas con la actividad 4 y otro para compartir "reflexiones finales" del curso.

\section{Análisis de la UA PIME}

La interacción entre experiencia y competencia que presentamos en esta sección se basa en el análisis de los foros asociados a las actividades 1,2 y 4 .

\section{Actividad 1. La experiencia: elemento clave en la resolución y evaluación de un diseño didáctico}

En esta actividad se pidió resolver, en parejas, el diseño didáctico propuesto por dos profesores en la actividad 4 del curso ADME I. Cada pareja resolvió y evaluó el diseño didáctico propuesto por otro dueto y elaboró una guía didáctica que explicitara, bajo sus propias interpretaciones: (1) las características del diseño didáctico, (2) los objetivos didácticos (qué es lo que se quiere enseñar a los estudiantes), (3) la actividad que se espera de los estudiantes, y (4) la actividad que se espera del profesor. Esto con el objetivo de que, si un profesor externo a este curso quisiera implementar dicho diseño con sus estudiantes, contara con elementos para hacerlo. Se habilitaron dos foros, el primero, titulado "Sobre la Actividad 1", para expresar dudas, reflexiones y comentarios acerca del desarrollo de esta actividad; el segundo (titulado "Reacción a la guía elaborada"), para presentar las guías didácticas de manera que la pareja de autores del diseño reaccionara a los comentarios de la pareja de evaluadores.

En el primer foro, los profesores expresaron dudas sobre las tareas propuestas en el diseño; algunas no estaban claramente descritas. En la figura 1 se muestra el diálogo entre dos profesores; la profesora Hм pide precisiones sobre el diseño didáctico en general y luego sobre tareas particulares, y el profesor sc le responde. 
Profesora HM. Estimados compañeros, Mucho agradeceré las siguientes precisiones a su propuesta: ¿Para qué nivel académico está orientada? ¿Cómo se pretende llegar a la gráfica descrita en la tarea 1 inciso c?, ¿cuáles son las unidades del eje de las abscisas que utilizaron?, pues las propuestas en el ejercicio no concluye en la misma gráfica. Para la tabla del inciso d) (misma tarea), ¿todos los valores de $V$ son positivos? pues ello no llevaría a la gráfica que proponen. Respecto a la tarea 2, ¿cuáles son las unidades del eje de las abscisas que utilizaron?, pues las funciones propuestas en el ejercicio llevadas a un graficador no nos lleva a la misma gráfica que ustedes proponen. En esa misma tarea no se explicita cómo desarrollar los cálculos para la tabla 4. Ello repercute en la definición de la tarea 3. Para la tarea 4, así como en toda la actividad, nos resulta confuso el uso de $\mathrm{Hz}$ como unidad de medida del sonido. Por el momento son nuestras dudas. [...]

Profesor JC. [...] 1. El nivel es bachillerato, últimos semestres. Los estudiantes deben conocer ecuaciones simultáneas, valor absoluto, promedio y matrices (hablamos del área de físico-matemáticas del último grado de bachillerato) 2. Las unidades de las ordenadas son, en términos generales "energía" en un sentido abstracto. Todos los senos tienen amplitud 1 (su coeficiente) para que las funciones originales no pasen de uno y se queden normalizadas. En la práctica, estas unidades pueden ser "unidades de volumen", "intensidad" o incluso "volts" si el sonido ya fue procesado por un micrófono. Es importante lo de normalizar a 1 para que la resonancia supere este valor cuando exista. 3. La tarea 1 inciso c involucra un poco de investigación. El objetivo es que el alumno sepa que la frecuencia de LA de una octava alta es el doble de la primera LA ( $440 \mathrm{~Hz}$ contra $880 \mathrm{~Hz}$ ) y que los sonidos de mayor frecuencia son más agudos. 4. Tiene razón. Los muestreos de la tabla (d) no son correctos. [...]

Figura 1. Extracto de la participación en el foro "Sobre la Actividad 1": profesores HM (mexicana) y лC (mexicano)

Esta interacción en el foro que muestra cómo resolver el diseño didáctico elaborado por otros constituye una primera evaluación del mismo (claridad, objetivos de las tareas, elementos contextuales involucrados, modelos matemáticos en juego, conocimientos disponibles, conocimientos por construir, pertinencia) y hace conscientes a los autores de los aspectos implícitos que la profesora $\mathrm{HM}$ considera necesarios detaIlar, por ejemplo, el nivel educativo para el que fue diseñado. También emergieron dudas relacionadas con el contexto educativo del país donde residen los profesores (véase la figura 2), que fueron atendidas con explicaciones que permitieron contextualizar el contenido del diseño. 
Profesor NG: Hola compañeros, buenos días.

Tengo una duda respecto a cómo se nombran los niveles educativos en los demás países, he visto liceo en algunos comentarios y entiendo es bachillerato; ciclo medio creo es secundaria. A ver si alguien aclara mi duda, pues me será útil para comprender sus comentarios en todos los cursos.

Como los trabajos a resolver para la actividad 1 corresponden a los de otro equipo, les envío como se denominan en México:

preescolar: hasta los 6 años de edad

primaria: de aprox 7 a 12

secundaria de 12 años a 15.

bachillerato: 15 a 18 aprox. También se le conoce como preparatoria, nivel medio superior, vocacional, bachillerato técnico.

Se le conoce como educación básica hasta el nivel bachillerato. Superior: licenciatura, universidad.

Saludos, gracias

Profesora CR. Hola [...]

En Uruguay la diferencia con ustedes es que nosotros Ilamamos secundaria a los 6 años que los alumnos cursan con edad entre 12 y 18 años, ésta se divide en Ciclo Básico $\left(1^{\circ}, 2^{\circ}\right.$ y $\left.3^{\circ}\right)$ y es lo que ustedes nombran como secundaria y en Bachillerato $\left(1^{\circ}, 2^{\circ}\right.$ y $\left.3^{\circ}\right)$ que ustedes lo llaman igual que nosotros.

Saludos

Profesor NG: Hola [...]

Muchas gracias, nunca me imaginé le llamaran secundaria a los seis años! Es que en los foros me confundo demasiado con los términos.

saludos.

Figura 2. Extracto de la participación en el foro Sobre la Actividad 1": profesores NG (mexicano) y CP (uruguaya)

Con esta primera actividad se pretende que los profesores experimenten la necesidad de convertir los diseños didácticos en recursos didácticos que puedan ser compartidos entre pares. En ello, se apeló a la experiencia de los profesores en cuanto a su conocimiento tanto de contenido matemático como pedagógico, así como aquella adquirida en la UA ADME I. En este sentido, las observaciones de los profesores variaban en términos de lo que cada uno consideraba necesario explicar, de manera que tuvieron la oportunidad de hacer trabajo colaborativo, de comprometerse con la evaluación del recurso y tomar decisiones propias respecto de qué cuestionar y qué pasar por alto. 
En la siguiente actividad, se les proporcionaron nuevos recursos basados en la investigación para tener puntos de referencia más compartidos en torno a los cuales evaluar el diseño. Estos recursos exigían interpretación y acción, por parte de los profesores, para alinear sus primeras observaciones sobre el recurso didáctico con las competencias que las herramientas teóricas exigían.

\section{Actividad 2. Competencia para el análisis ergonómico de recursos didácticos}

Los educadores elaboraron un documento denominado "Herramientas teóricas para el análisis de recursos didácticos", que sintetizaba elementos teóricos de la tesis doctoral de Georget (2009). Los profesores leyeron el documento y con base en él se les pidió: (1) describir las características generales de las herramientas, y (2) analizar su utilidad, la importancia de conocerlas y la forma en que podrían ser utilizadas. Se habilitó un foro en equipos (cuatro equipos de cinco integrantes) para reflexionar sobre la lectura. Para complementar información sobre las herramientas teóricas, se colocó un video producido por Georget para explicar las herramientas teóricas. Este fue un recurso que, junto con el documento, apoyó la competencia.

Las herramientas presentadas son categorías de análisis de los recursos didácticos (utilidad, utilizabilidad, adaptabilidad y aceptabilidad) y de los diferentes potenciales de las actividades abiertas y de investigación (de investigación, de resistencia y de resistencia dinámica, de debate y didáctico). La utilizabilidad, por ejemplo, permite analizar el tiempo necesario para que el profesor consulte el recurso didáctico y pueda evaluar su interés para la práctica. Con esta categoría se relaciona la adaptabilidad, que consiste en que el profesor pueda adaptar el recurso didáctico a su propia práctica y personalizarlo. Los potenciales son mucho más complejos, por ejemplo, el potencial de investigación de un recurso didáctico debe permitir a los estudiantes, más que aplicar técnicas enseñadas, proponer soluciones para un problema nuevo, implicarse en la investigación: cuestionar, activar su curiosidad, validar los pasos realizados y asegurarse de que la solución propuesta es la adecuada.

Las interacciones en el foro resultaron sumamente interesantes, pues muestran cómo los profesores fueron produciendo y adoptando las categorías de análisis como un conocimiento más común con el cual poder evaluar la ergonomía de los recursos didácticos (véase la figura 3), antes analizados bajo sus propias interpretaciones (actividad 1). 
Profesora HM. [...] Cuando partimos de la evaluación para los recursos desarrollados el curso anterior, me surgieron dudas respecto a ¿qué elementos debo considerar para evaluar el recurso? ¿Con qué autoridad puedo hacerlo? ¿Es apropiado mi comentario o no? [...] creo que con las categorías de análisis que proporciona el autor, puedo entender que los recursos a evaluar no deben centrarse, únicamente, en mis gustos, preferencias o nivel académico que trabajo. Existen categorías como la UTILIDAD, que va de la mano con el referente académico, pero también con la ACEPTABILIDAD, pues aunque ésta última responde a factores más subjetivos como la cultura y los valores sociales, esto mismo hace que un grupo pueda aceptar como útil el recurso. Esta aceptación se logra a partir de los intereses sociales. [...]

Figura 3. Extracto de la participación en el foro "Reflexiones sobre las herramientas teóricas": profesora HM (mexicana)

La profesora HM reconoce que evaluar un recurso didáctico bajo sus propias interpretaciones no es suficiente, ella encontró en las herramientas proporcionadas nuevas perspectivas (véase la figura 3). La actividad motivó la experiencia e iniciativa de los profesores, les ayudó a emitir juicios y hacer evaluaciones mutuas; las herramientas teóricas les permitieron imaginar puntos fijos en torno a los cuales coordinar esos juicios y evaluaciones, lo cual se concretaría en la siguiente actividad.

\section{Actividad 4. La alineación y sus alcances}

En el foro de reflexiones finales fue posible observar cómo los profesores fueron viviendo el proceso de crear un diseño didáctico, someterlo a juicio y evaluación entre pares y alinear sus interpretaciones prácticas con unas perspectivas más teóricas para crear instrumentos con mejores posibilidades de compartirlas con sus pares. Por ejemplo, la profesora $\mathrm{CP}$, en el foro de "Reflexiones finales" detalla la vivencia de las distintas actividades realizadas y reconoce el potencial del trabajo colaborativo (véase la figura 4).

[...] El desarrollo del curso lo viví como un proceso que me permitió ir repensando y analizando una secuencia de actividades, lo que me llevó a analizar algunas de las actividades que planteo a mis alumnos. Creo que quedó en evidencia lo rico e importante que es discutir y analizar con otros colegas las actividades que Ilevaremos o hemos Ilevado al aula, esto nos posibilita distinguir elementos de las mismas que de otra manera no consideraríamos.

$[\ldots]$

Figura 4. Extracto de la participación en el foro "Reflexiones finales": profesora CP (uruguaya) 
Las acciones de algunos profesores trascendieron el contexto educativo donde laboran. Ellos vivieron experiencias de alineación no solo con los recursos proporcionados por el campo de la investigación en educación matemática, sino con los de su institución. Las actividades de esta uA los situaron en el reconocimiento de sus propias actividades, las que suelen implementar en el día a día de su enseñanza; en ello, cada profesor produjo un significado particular, de acuerdo con sus propios intereses y necesidades. Los profesores adoptaron no solo los nuevos elementos de conocimiento proporcionados (herramientas de ergonomía y potenciales de las actividades abiertas y de investigación), sino también formas de llevarlos a su contexto. Este es el caso del profesor Jc, quien comentó haber puesto en acción ese conocimiento (véase la figura 5).

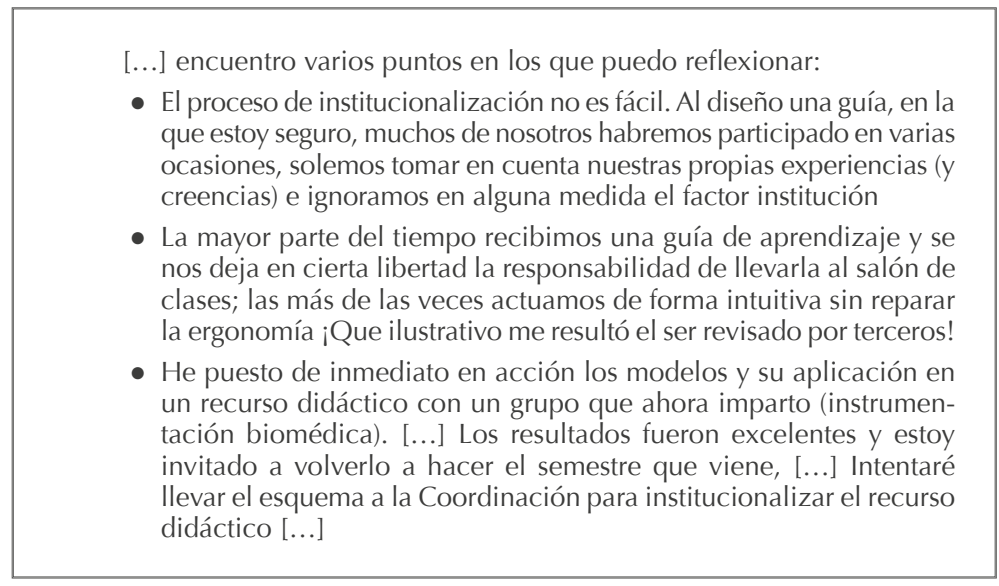

Figura 5. Extracto de la participación en el foro "Reflexiones finales': profesor Jc (mexicano)

Reconocer el poder de alineación que tiene la institución educativa es necesario en la medida en que ello favorece el proceso de identidad. Para Wenger (2001), "definimos quiénes somos negociando maneras locales de pertenecer a constelaciones más amplias y de manifestar estilos y discursos más amplios" (p. 188). La idea del profesor JC sobre "Ilevar el esquema a la Coordinación para institucionalizar el recurso didáctico" ejemplifica una forma de contribuir a su propia institución proponiendo un recurso didáctico que fue producto de un proceso más amplio en el que adoptó discursos y estilos tomados de la investigación en educación matemática. De esta manera, se puede apreciar que el profesor no hizo una aplicación fija de esos discursos y estilos, sino que al tener en cuenta su propia práctica e institución los adoptó a su contexto.

En esta UA, el diseño y la evaluación de un recurso didáctico invitaron a los profesores a compartir sus propios diseños, a pensar el proceso que implica la elaboración y evaluación de los recursos didácticos que suelen 
emplear, a verse a sí mismos como parte del proceso de institucionalización del conocimiento matemático en el contexto escolar. Estos elementos nos parecen fundamentales para sensibilizar a los profesores sobre la importancia de llevar a sus estudiantes a ser parte de una comunidad de estudio, en la que ellos y el profesor conjuntamente "institucionalizan" conocimiento matemático. Esta sensibilización se sustenta en la reflexión conjunta con sus pares y con los educadores, así como en la vivencia de haber sido un productor de recursos didácticos y no solo un consumidor que sigue un conjunto de instrucciones.

\section{Conclusiones y reflexiones finales}

En este escrito se han presentado los resultados del análisis de una UA concebida e implementada en un programa de desarrollo profesional en la modalidad en línea y a distancia. En el diseño de esta UA se ha tenido por objetivo proponer herramientas teóricas y metodológicas de la educación matemática para el análisis de recursos didácticos del y para el profesor de matemáticas. La experiencia de los profesores de matemáticas ha sido considerada como base para proponer los recursos de aprendizaje que representan la competencia establecida por los educadores. La forma de considerar la experiencia de los profesores en el diseño e implementación de la UA nos parece que es de equidad ya que:

» En nuestros cursos no hay distinción de género, país de procedencia o nivel educativo. Por el contrario, estos elementos se consideran como parte de la experiencia del profesor de matemáticas. Es decir, la heterogeneidad del grupo es indispensable porque forma parte de la trayectoria de aprendizaje de cada profesor, la cual hace que su experiencia sea única e indispensable.

»Se reconoce al profesor como un miembro de la comunidad del Prome. No hay relaciones de poder, sino de compromiso mutuo. Ambos aprendemos, los profesores complementan la práctica de los educadores, y estos, la de ellos.

»Se reconoce el conocimiento práctico del profesor como legítimo para organizar su propio aprendizaje. Los profesores tienen oportunidad de intervenir en su aprendizaje y tomar decisiones que influyan en su práctica docente, de acuerdo con sus propias necesidades.

"Al ofrecerles a los profesores recursos para coordinar su aprendizaje y comprometerlos a participar en la producción y adopción del significado de esos recursos, se brinda la posibilidad de que cada uno de ellos se sienta reconocido. Por un lado, tienen la oportunidad de 
comprenderse a sí mismos como profesores aprendices; por otro, de hacer intervenir su capacidad para innovar su práctica, en consecuencia, su propia identidad.

Nuestra propuesta de hacer interactuar la experiencia y la competencia parece un elemento indispensable para fomentar la equidad en el aprendizaje de los profesores. Sin embargo, en los cursos no se discute de manera directa cómo pueden ellos fomentar esa equidad en sus clases. Lo que sí creemos es que el tipo de actividades que los profesores enseñan pueden ser medios importantes para fomentar la equidad. Esas actividades se asemejan a las que realizan aquí. En las actividades que los profesores propongan se esperaría que los estudiantes fueran vistos como parte de una comunidad de estudio, capaces de investigar y de estudiar cuestiones complejas, provenientes de contextos reales y donde el uso de las matemáticas tenga efectos sobre su persona y medio social. Es decir, hacer del estudio de las matemáticas una forma de comprenderse a sí mismos y de reconocerse como parte del mundo.

\section{Referencias}

Artigue, M. (2011). Les défis de l'enseignement des mathématiques dans l'éducation de base (fr). París:unesCO.

Chevallard, Y. (2013). Enseñar matemáticas en la sociedad de mañana: Alegato a favor de un contraparadigma emergente. Journal of Research in Mathematics Education, 2(2), 161-182.

Dewey, J. (1920). Reconstruction in philosophy. Nueva York: Holt.

Doerr, H. M. (2007). What knowledge do teachers need for teaching matehmatics through applications and modelling? En W. Blum, P. L. Galbraith, H-W. Henn y M. Niss (eds.). Modelling and Applications in Matemathics Education. The 14th ICMI Study. USA: Springer

Georget, J-P. (2009). Activités de recherche et de preuve entre pairs à l'école élémentaire: perspectives ouvertes par les communautés de pratique d'enseignants. París: IREM de París.

Moreno B., Ma. G. (2003). El posgrado para profesores de educación básica (cuadernos de discusión n. ${ }^{\circ}$ 5). México: Secretaría de Educación Pública.

Organización de las Naciones Unidas para la Educación, la Ciencia y la Cultura,unesCo. (1999). Declaración sobre la ciencia y el uso del saber científico. [En línea]. París. Recuperado de http://www.unesco.org/ science/wcs/esp/declaracion_s.htm

Sowder, J. T. (2007). The mathematical education and development of teachers. En F. K. Lester, Jr. (ed.). Second handbook of research on mathematics teaching and learning (pp. 157-223). Charlotte, NC: Information Age. 
Weissglass, J. (1997). Ripples of hope: Building relationships for educational change. Santa Barbara, CA: Center for Educational Change in Mathematics and Science.

Wells, G. (2004). Dialogic inquiry. Toward a sociocultural practice and theory of education. Nueva York: Cambridge University Press.

Wenger, E. (1998/2001). Comunidades de práctica. Aprendizaje, significado e identidad (G. Sánchez Barberán, trad.). Barcelona: Paidós. [Reimpreso de Communities of practice. Learning, meaning, and identity, 1998, Cambridge, Reino Unido: The Press Syndicate of the University of Cambridge].

Wenger, E. (2010). Communities of practice and social learning systems: The career of a concept. En C. Blackmore (ed.). Social learning systems and communities of practice (pp. 179-199). Nueva York: Springer. 